\title{
A small, novel, remote in-vessel inspection system for the Alcator C-Mod tokamak
}

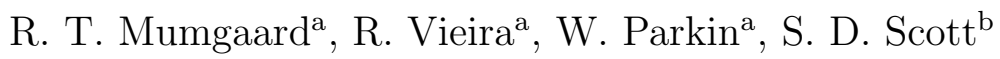 \\ ${ }^{a}$ MIT Plasma Science and Fusion Center \\ ${ }^{b}$ Princeton Plasma Physics Laboratory
}

Abstract

A small robust system has been constructed for in-situ visual inspection of the Alcator C-Mod tokamak. The system consists of a small, light, wide-angle high definition camera and LED package housed in a nacelle on the end of thin, rigid, 3.5 meter long support pole. The nacelle has two actuated degrees of freedom allowing the camera to observe nearly $4 \pi$ steradians. The support pole has a specific slight curve that allows it to pass to either side of the center column of the tokamak to observe the entirety of the vessel interior, while still fitting through the small aspect ratio Alcator C-Mod vacuum port structure. The support pole and camera can enter the vessel through any horizontal vacuum port with an inner diameter greater than $4 \mathrm{~cm}$, thus a dedicated port is not required. The inspection is typically undertaken during maintenance periods when the vessel is filled with a noble gas near atmospheric pressure thus minimizing the influx of water vapor and the concomitant loss of wall conditioning. The system is operated manually, producing photos and video which are reviewed in near real-time. Nearly the entire vessel, including the plasma facing components, can be carefully inspected in 3 to 5 hours. The system provides improved characterization of the interior components and 
surfaces of the tokamak with a modest engineering and operational effort. Information gathered from the system has identified damage to plasma facing components that were interfering with tokamak operation, as well as damage to mechanical components which were redesigned during the remainder of the campaign, thereby enhancing program planning.

Keywords: inspection, tomakak, remote handling, cameras, Alcator C-Mod

The high vacuum, radiation, and temperature environment inside modern fusion experiments precludes frequent manned-access for inspection. Therefore, a means to inspect the interior of fusion devices carefully and remotely in a manner similar to what is used in other hazardous environments[1] has long been recognized as being advantageous for operational monitoring and program planning. This is particularly important for future burning plasma experiments including both ITER and a demonstration power-plant that will require high availability while precluding manned entry[2]. Several groups have developed elaborate robotic systems with many degrees of freedom for in-situ inspection and repair in ultra-high vacuum and full magnetic fields for existing devices $[3,4,5]$, particularly for tokamaks with long pulse and component test programs. However, development and operation of similar large and complicated systems is often out of the scope for medium and small fusion experiments where inspection - a few times during a campaign - could still provide useful data for operational planning, engineering, and plasma surface science. This paper details a small, economical, and flexible, inspection system developed for and deployed on the Alcator C-Mod tokamak. 


\section{Motivation and design constraints}

Alcator C-Mod is a compact $(R=68 \mathrm{~cm}, a=22 \mathrm{~cm})$, high field $(<9 \mathrm{~T})$, normal-conducting tokamak[6]. Unlike many other tokamaks, C-Mod's diagnostics, limiters and heating antennas are bolted to structures inside the vacuum vessel rather than inserted through ports or operating through windows. This results in a large number of highly engineered components inaccessibly installed inside the vessel. These in-vessel components can be damaged during disruptions due to the high field, large plasma current and large stored thermal energy. Typically, the device is operated for $\sim 6-9$ months; the remainder of the year is used for manned-access inside the vessel to repair damaged components and install upgrades.

C-Mod's high power densities can lead to melting of the molybdenum and tungsten plasma-facing components[7]. Typically this melting is documented only when components are removed during manned-access at the end of a campaign. However, determining which events caused the most significant damage is important for understanding the plasma material interaction. Occasionally the plasma facing components are deformed significantly due to melting, requiring the plasma's magnetic configuration to be modified to prevent further damage and to allow reliable operation. Finally, sacrificial coatings have been deposited on various components to monitor material erosion due to plasma exposure. In addition to measuring these films after they are removed during a manned access period, it is desirable to visually monitor them at multiple times during the experimental campaign.

Fielding permanently installed machine protection cameras requires specialized cameras or the development of complex endoscopes due to Alcator 
C-Mod's high magnetic field, high heat flux, high neutron flux and strong accelerations during plasma disruptions. Additionally, the compact nature of the device, large toroidal field coils, external support structure and cryostat require the small number (10) of horizontal ports to be long radially and narrow toroidally $(\sim 1 \mathrm{~m}$ by $\sim 0.1 \mathrm{~m}$ ) with smaller vertical ports with similar aspect ratio. Therefore, unlike other tokamaks[8], only a small toroidal field of view is available from each of the limited number of protection cameras leaving much of the tokamak interior unmonitored. Furthermore, the lack of a dedicated illumination source limits the observational periods to operation with a plasma.

Therefore, there is a need to quickly view the entirety of the interior of the tokamak using a remote inspection system between manned-access maintenance periods. Such a system can be quickly deployed to inspect all the plasma facing components and sacrificial coatings. The system can provide important information about the condition of the materials that can then be used to optimize plasma operation. Additionally, observation of the condition and failure mechanisms of in-vessel components during an experimental campaign allows the engineering team to begin work on solutions immediately. During the subsequent manned-access maintenance period these solutions are installed.

\section{System design}

In contrast to more complicated inspection systems[9], this system is dedicated to infrequent visual inspections (occurring 1-3 times during a campaign) which allows the system to be small and purpose-built. Because C- 
Mod does not use radioactive fuels and because its magnets are energized only during plasma operation, unlike typical superconducting facilities, the vessel can be quickly approached and brought to atmospheric pressure. This is routinely done during an experimental campaign to allow changes to the vessel configuration such as adding or changing port mounted diagnostics. Therefore, the inspection system is not intended for operation in high vacuum, greatly simplifying the design[10]. However, to ensure cleanliness, the system forms a rudimentary seal to the tokamak to prevent air ingress. Furthermore, because the Alcator C-Mod vacuum configuration changes frequently the system is designed to utilize a variety of horizontal ports dependent on the diagnostic complement that is currently installed; it requires only a port with an inner diameter greater than $4 \mathrm{~cm}$ rather than multiple large dedicated ports[4].

The inspection system consists of an articulated nacelle containing the camera and lighting fixture that is mounted on the end of a $2.5 \mathrm{~cm}$ diameter, 3.5 meter long rigid stainless steel support pole. The pole has a slight, prescribed curve in the horizontal plane so when it is inserted into the tokamak it curves away from the central column while still fitting through the long and narrow horizontal ports. This allows the camera nacelle at the end of the pole to avoid the central column, moving past it to observe the far side of the tokamak. The pole can then be flipped 180 degrees about its axis and reinserted, missing the central column on the opposite side of the tokamak. These two trajectories, the articulated nacelle, and the wide camera field of view enable the in-vessel tokamak components and all plasma facing components to be viewed from a single entry port. The system inserted into the 


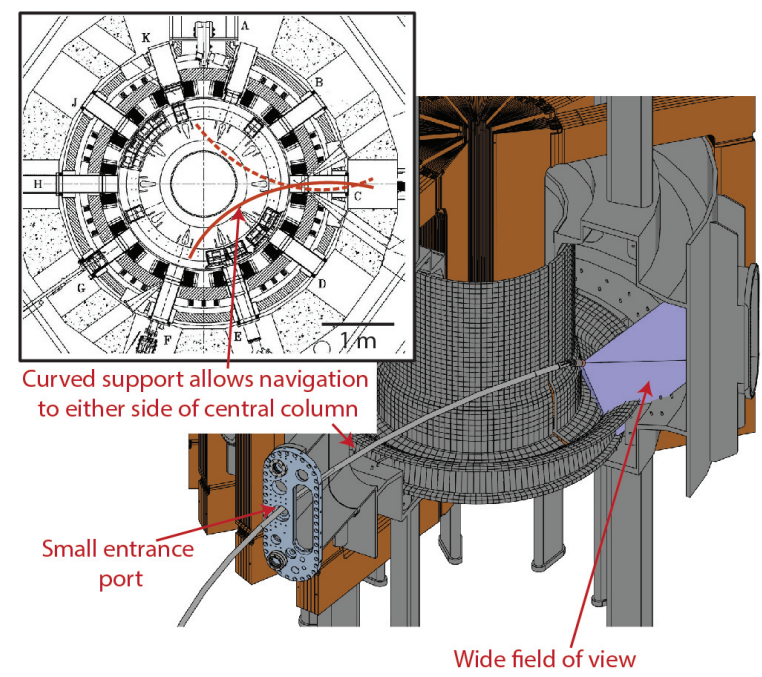

Figure 1: The inspection system consists of a rigid pole that is inserted through a horizontal vacuum port as shown in (a). The slight curve in the pole allows the system to avoid the center column. The pole can be inserted twice, avoiding the center column to each side to enable the nacelle to observe the entire tokamak utilizing only a single small port as shown in (b). The camera can view its entire surroundings at each point along each of the two trajectories, thus enabling inspection of the entire vessel main volume.

tokamak is shown in Figure 1.

Mounted in the copper and stainless steel nacelle is a small USB controllable consumer CMOS web camera. The camera has digital zoom capability, adjustable focus and is capable of 1280x720 high definition video operation and 5MP photography with a wide field of view (75 degrees diagonal). The camera has auto-exposure compensation to enable operation in low light conditions. The camera has been disassembled to yield only the lens assembly and an electronics chip which are small, $<2.5 \mathrm{~cm}$ diameter, and light.

At the front of the nacelle is a set of eight high intensity miniature $(4.6 \mathrm{x}$ $3.2 \mathrm{~mm}$ ) LEDs mounted in a ring on a printed circuit board to provide light- 
ing. The camera views through a hole in the center of the circuit board. The LEDs produce a total of 1400 lumens of white light with a wide illumination pattern. The circuit board is mounted to the copper portion of the nacelle, which acts as a heat sink for the LEDs in low conductivity atmospheres and includes a thermocouple to monitor the temperature. The LED assembly is recessed in the nacelle to provide protection from incidental collisions. The entire nacelle weighs $<0.3 \mathrm{~kg}$, allowing the support pole to be slim and light. The adjustable camera focus and variable lighting intensity allows metallic components between 0.1 and $1 \mathrm{~m}$ from the camera to be imaged accurately.

Two degrees of actuation allow the camera to fully observe its surroundings from the end of the pole. The nacelle is attached to the nacelle holder via a hinge that allows it to pivot 145 degrees from the pole axis in one direction, allowing the camera to look "backwards" towards the entrance port. A cable-in-conduit is used to control this pitch angle of the nacelle, and a torsion spring returns the nacelle to the straight position. The nacelle and nacelle holder are attached to the end of a corrugated metal tube that is inserted inside the curved rigid support pole. A schematic of the system is shown in Figure 2. This tube runs the entire length of the rigid pole. The tube's outer diameter is slightly smaller than the inner diameter of the rigid pole allowing it to rotate $>360$ degrees inside the rigid pole. The cable-in-conduit, USB cable and LED power cables are routed inside the corrugated tube and rotate with it. The corrugation allows the tube to transmit torsion while still being flexible enough to conform to the slight curve in the rigid pole as it rotates. A lever assembly is affixed to the air-side end of the corrugated tube, thus rotation of this assembly about the pole axis on the air-side rotates the nacelle 
assembly about the pole axis on the tokamak-side. The lever can be pivoted 45 degrees relative to its assembly to pull the cable-in-conduit to control the pitch of the nacelle. The friction between the corrugated tube and rigid pole along with a friction brake on the lever assembly ensures the nacelle's roll and pitch, respectively, stay in the same pose even when the lever is not being held. The combination of pitch and roll and the camera's wide field of view enables the camera to observe $\sim 4 \pi$ steradians solid angle from the pole end using a single, simple, manually-controlled lever. Since the rotation of the lever assembly is 1:1 with the rotation of the nacelle and the angle of the lever is proportional to the pitch of the nacelle, the position of the camera relative to the tokamak-side of the rigid pole is readily apparent from the position of the lever relative to the air-side of the rigid pole. Photographs of the system are shown in Figure 3.

\section{Inspection procedure}

The simplicity of the system allows for it to be quickly deployed with little preparation. A plasma diagnostic near the tokamak mid-plane on a horizontal flange is typically removed from its gate valve to allow the inspection system to be inserted into the tokamak. A support guide structure is bolted to the port flange to stabilize the inspection system and to protect the port flange and gate valve. The inspection system is both cleaned and is contained in a flexible plastic envelope that forms a rudimentary seal to

the air-side end of the pole. The gate valve is opened and the pole is slowly inserted while the camera is used to observe the interior of the tokamak in real-time on a computer monitor. The envelope is purged of air after be- 


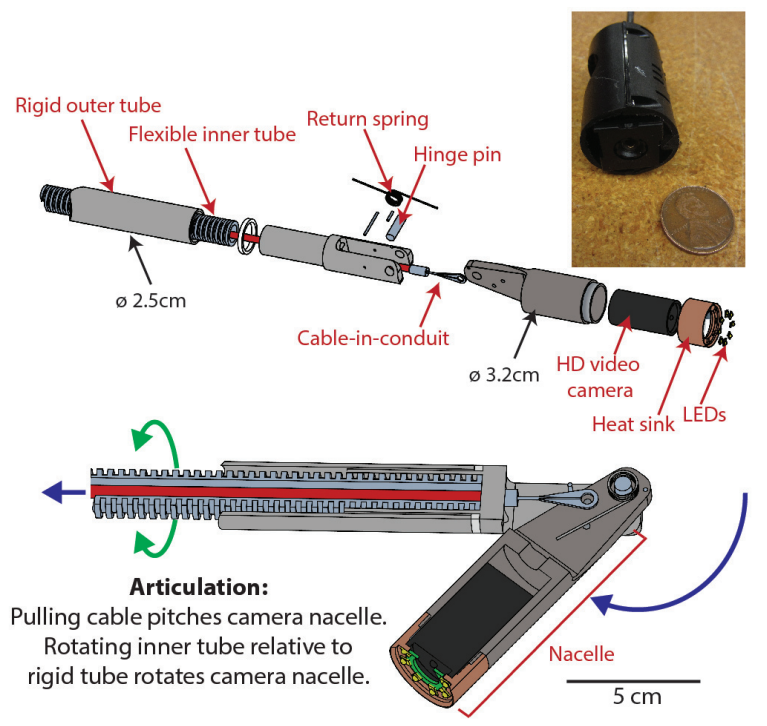

Figure 2: The small inspection system contains a small consumer web camera (inset) and LEDs at the end of the support pole. Two degrees of freedom, pivoting the camera containing nacelle using a cable-in-conduit and rotating the flexible corrugated inner tube relative to the rigid support pole, allow the camera to observe its surroundings. 

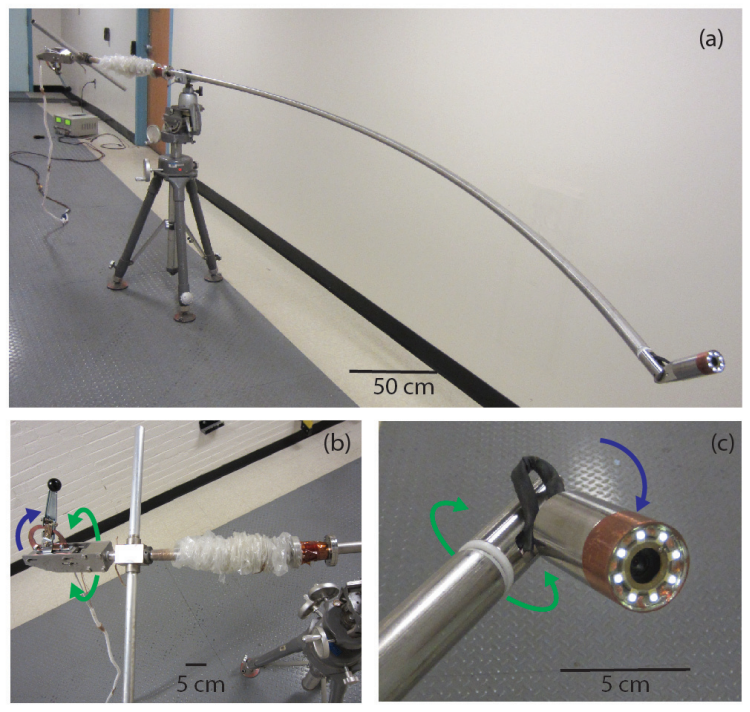

Figure 3: Photographs of the inspection system that show the system with the flexible plastic envelope and vacuum flange pulled back. The curve in the support pole is apparent (a). The control lever on the air-side pole end (b) controls the position of the camera and LED containing nacelle on the invessel pole end (c). The pitch of the nacelle relative to the pole axis is actuated by pivoting the lever in its assembly (blue arrows), and the roll of the nacelle relative to the pole axis is controlled by rotating the entire lever assembly about the pole axis (green arrows). 
ing affixed to the vacuum port and the tokamak is brought from vacuum to slightly above atmospheric pressure using an inert gas, typically dry argon or helium, to ensure that air is not drawn into the vessel via any leaks in the envelope. The flexible envelope bunches up at the vacuum port as the clean, bare pole is inserted further into the tokamak. The inspection process typically takes $3-5$ hours and is completed in parallel with other maintenance activities, such as the installation or maintenance of diagnostics.

The system is designed to be well-balanced as it is inserted into the tokamak and the orientation of the rigid pole is controlled using two long handles fixed to the air-side pole end. That fact that it is light weight $(<10 \mathrm{kgs})$ and has a support guide structure allows for guidance by hand. The inspection process typically involves three operators: one person to operate the actuator, moving the nacelle and navigating the space while observing the camera output in real-time; one person to operate the camera control computer, recording video, taking photographs and making notes; and one person to guide and stabilize the pole position and to operate the gate valve. The operators are chosen from the team responsible for work inside the tokamak during manned access maintenance periods and thus have a working knowledge of the interior configuration. This both aids navigating the camera and identifying important parts of the tokamak to document. The support guide structure and port geometry restricts the range of movement of the pole, though the system can still come into contact with interior surfaces. This is avoided by slow and deliberate operation by trained operators. During one nacelle did contact the inner wall and lower divertor, but no damage resulted beyond small surface scratches to the molybdenum tokamak surface and the 
stainless steel camera nacelle. Care was taken during design to use captured components to prevent the dropping of any of the system's components in the vessel. Additionally, the entire assembly is streamlined to prevent the system from becoming caught on any interior components.

Typically a survey of the entire tokamak is completed first by observing across the full actuation range with the pole inserted at different depths into the tokamak, passing to each side of the center column once. Photos and video are uploaded onto the computer network so others can observe the inspection progress in near real-time. The wide field of view and ample lighting enable the identification of antennas and other distinct structures in a given photo, allowing the observer to orient themselves. Furthermore, the photos are labeled by the computer operator to indicate the position from which they were taken (i.e. the position of the camera in the tokamak). Areas of interest are identified prior to the inspection and during this survey and are then revisited and photographed in detail from many different angles while notes are taken.

Since the camera can observe its entire surroundings at each insertion depth, the majority of the vessel except for the wall opposite the entrance port can be inspected at a distance $<1$ meter from multiple angles by observing across the full actuation range from multiple points on the trajectory. The entirety of the tokamak main chamber surfaces can be observed at distances $<1.5$ meter from at least one angle. However, the system can only observe down a 7 of 10 of the horizontal ports and very few of the vertical ports from a given entrance port. Typically the port used for inspection is slightly over sized, and the support guide allows the entire pole to be piv- 
oted upward and downward. This brings the camera closer to items off the mid-plane allowing closer inspection of the plasma-facing components in the divertor. (note: this close inspection is only feasible for $\sim 50 \%$ of the plasma facing components from any given port.)

Once the vessel has been photographed satisfactorily, the system is removed, the gate valve is closed, the support guide is un-bolted, and the removed plasma diagnostic is reinstalled. The tokamak is pumped down to vacuum and plasma operation can resume almost immediately. Typically inert-gas fill maintenance periods are completed on Fridays to allow electron cyclotron discharge cleaning (ECDC) over the weekend. Full-length researchgrade plasmas with little increase in the hydrogen fraction or plasma impurity content are obtained on the first attempt after an inspection and a period of ECDC. The photos and video are then used to inform further operational constraints, gauge the condition of the plasma facing components and sacrificial coatings, and to inform preparation for any required repairs.

\section{Examples of use}

First operation to provide information about the condition of the interior of the vessel was in Fall 2009, and the system has been used on two occasions during the middle of an experimental campaign. The system has also been used to inspect the tokamak at the end of an experimental campaign immediately before manned-access, documenting the vessel state before any coatings are disturbed due to human contact. Several examples of observations using the system are shown in Figure 4.

Panel (a) shows a damaged set of tungsten tiles in the divertor, the obser- 

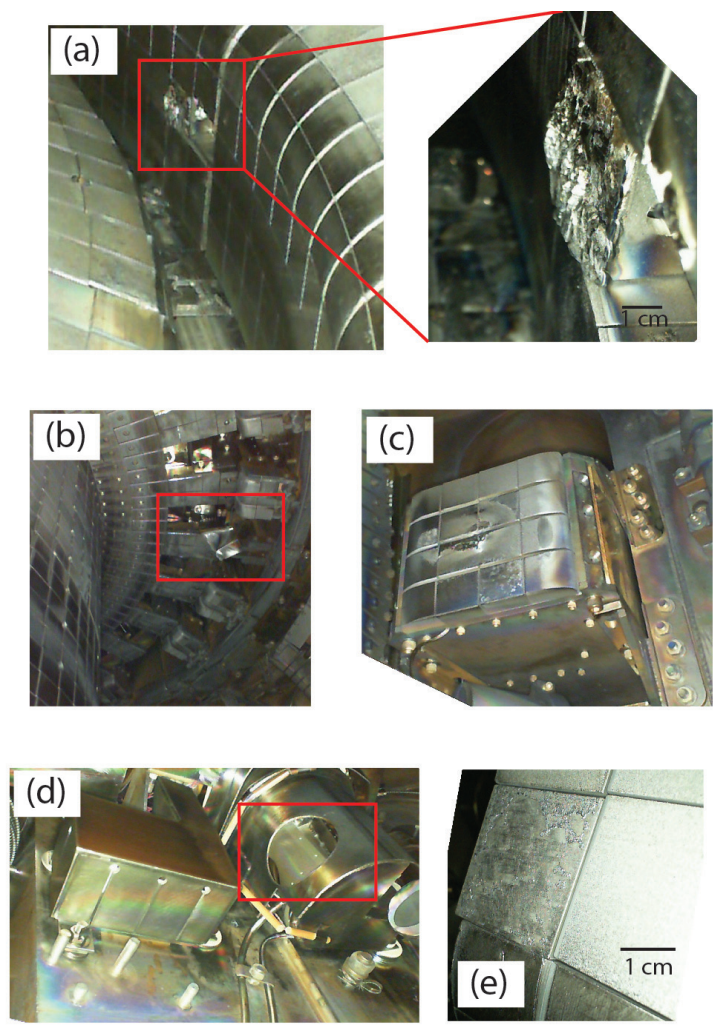

Figure 4: Examples of photos taken by the inspection system to document the vessel condition and inform operations and maintenance plans. 
vation of which confirmed spectroscopic evidence of severe and progressive melting near the typical plasma strike point location. The photographs and video informed operational changes to allow reliable operation without further divertor damage. The experience with the divertor melting in this case led to increased understanding of the plasma material interaction[7]. Careful inspection with the system led to identification of the failure mode and to a redesign of divertor components. This redesign was undertaken during the remainder of the campaign, and the new design was ready in time for implementation at the next manned access maintenance period. Panel (b) shows a gas baffle failure in the upper divertor (highlighted in red) whose identification and avoidance enabled cleaner and more reliable plasma operation. Panel (c) shows fast-ion damage to the outer midplane limiter, the timely identification of which allowed the damaging event to be better identified which informed avoidance procedures. Panel (d) shows a failed mechanical shutter for the Motional Stark Effect (MSE) diagnostic (highlighted in red). The photos from the inspection system allowed the failure mode to be identified. As a result, a more robust replacement shutter was designed and was ready for installation at the next manned-access opening. Panel (e) shows images which documented the state of tiles with a sacrificial boron coating. Visual documentation of these tiles during the campaign provided better understanding of the changes in surface condition, adhesion and uniformity due to plasma exposure. Visual inspection of boron-nitride coated antenna Faraday rods has also been conducted[11]. 


\section{Conclusion}

A novel, small, flexible remote inspection system has been developed and deployed on the Alcator C-Mod tokamak facility. This system allows the interior of the compact device to be viewed during a short maintenance period. The system is inserted through a small vacuum port into the tokamak while it is slightly over-pressurized with a noble gas. The articulated high definition camera and LED package can be steered remotely to observe the entire interior, including plasma facing components from the end of the speciallycurved rigid support pole. The simplicity of the design allows the vessel to be quickly inspected on short notice, providing information that is used to inform operation, physics, and planning with little impact to the cleanliness of the vacuum.

\section{Acknowledgments}

This work was supported by US DOE award DE-FC02-99ER54512.

[1] Y. Perrot, L. Gargiulo, M. Houry, N. Kammerer, D. Keller, Y. Measson, G. Piolain, A. Verney, Long-reach articulated robots for inspection and mini-invasive interventions in hazardous environments: Recent robotics research, qualification testing, and tool developments, Journal of Field Robotics 29 (1) (2012) 175-185. doi:10.1002/rob.20422.

URL http://doi.wiley.com/10.1002/rob. 20422

[2] Y. Perrot, J. J. Cordier, J. P. Friconneau, L. Gargiulo, E. Martin, J. D. Palmer, A. Tesini, ITER articulated inspection arm (AIA): r\&d progress on vacuum and temperature technology for remote 
handling, Fusion Engineering and Design 7579 (2005) 537-541. doi:10.1016/j.fusengdes.2005.06.028.

URL http://www.sciencedirect.com/science/article/pii/S0920379605001134

[3] L. Gargiulo, P. Bayetti, V. Bruno, J. Hatchressian, C. Hernandez, M. Houry, D. Keller, J. Martins, Y. Measson, Y. Perrot, F. Samaille, Operation of an ITER relevant inspection robot on tore supra tokamak, FUSION ENGINEERING AND DESIGN 84 (2-6) (2009) 220-223.

[4] X. Peng, Y. Song, C. Li, M. Lei, G. Li, Conceptual design of EAST flexible in-vessel inspection system, Fusion Engineering \& Design 85 (79) (2010) 1362-1365. doi:10.1016/j.fusengdes.2010.03.043.

[5] X. Peng, J. Yuan, W. Zhang, Y. Yang, Y. Song, Kinematic and dynamic analysis of a serial-link robot for inspection process in EAST vacuum vessel, Fusion Engineering \& Design 87 (5/6) (2012) 905-909. doi:10.1016/j.fusengdes.2012.02.041.

[6] I. Hutchinson, H. Becker, P. Bonoli, N. Diatchenko, S. Fairfax, C. Fiore, R. Granetz, M. Greenwald, D. Gwinn, S. Hakkarainen, The physics and engineering of alcator c-mod.

[7] J.W. Coenen, K. Krieger, B. Lipschultz, R. Dux, A. Kallenbach, T. Lunt, H.W. Mueller, S. Potzel, R. Neu, A. Terra, Evolution of surface melt damage, its influence on plasma performance and prospects of recovery, Journal of Nuclear Materials 438 (Supplement) (2013) S27-S33. doi:10.1016/j.jnucmat.2013.01.005. 
[8] J. Chung, D. C. Seo, In-vessel visible inspection system on KSTAR, Review of Scientific Instruments 79 (8) (2008) 083504. doi:10.1063/1.2969654.

[9] M. Houry, L. Gargiulo, C. Balorin, V. Bruno, D. Keller, H. Roche, N. Kammerer, Y. Measson, F. Carrel, V. Schoepff, Diagnostics carried by a light multipurpose deployer for vacuum vessel interventions, FUSION ENGINEERING AND DESIGN 86 (9-11) (2011) 1868-1871.

[10] J.-B. Izard, L. Gargiulo, D. Keller, Y. Perrot, Hardening inspection devices to ultra-high vacuum, temperature and high magnetic field, IEEE Transactions on Applied Superconductivity 20 (3) (2010) 1767-1772. doi:10.1109/TASC.2010.2040607.

[11] Y. Lin, J. Irby, B. Lipschultz, E. Marmar, D. Whyte, S. Wolfe, S. Wukitch, Hydrogen control in alcator c-mod walls and plasmas, Journal of Nuclear Materials 363 920-924. doi:10.1016/j.jnucmat.2007.01.115. 
